\title{
Probing latitudinal variations of the solar magnetic field in cycles 21-23 by Parker's Two-Layer Dynamo Model with meridional circulation
}

\author{
E. Popova ${ }^{1}$, V. Zharkova ${ }^{2}$, and S. Zharkov ${ }^{3}$ \\ ${ }^{1}$ Department of Physics, Moscow State University, Lebedev strt. 1, Moscow, 119991, Russia \\ ${ }^{2}$ Department of Mathematics and Information Systems, Northumbria University, Newcastle upon Tyne, NE2 1XE, UK \\ ${ }^{3}$ Department of Physics and Mathematics, University of Hull, Kingston-upon-Tyne, HU6 7RS, UK \\ Correspondence to: V. Zharkova (valentina.zharkova@northumbria.ac.uk)
}

Received: 13 March 2013 - Revised: 6 October 2013 - Accepted: 15 October 2013 - Published: 20 November 2013

\begin{abstract}
Principle component analysis (PCA) of the solar background magnetic field (SBMF) measured from Wilcox Solar Observatory (WSO) magnetograms revealed the following principal components (PCs) in latitudes: two main symmetric components, which are the same for all cycles 21-23, and three pairs of asymmetric components, which are unique for each cycle. These SBMF variations are assumed to be those of poloidal magnetic field travelling slightly offphase from pole to pole while crossing the equator. They are assumed to be caused by a joint action of dipole and quadruple magnetic sources in the Sun.

In the current paper, we make the first attempt to interpret these latitudinal variations in the surface magnetic field with Parker's two-layer dynamo model. The latitudinal distributions of such waves are simulated for cycles $21-23$ by the modified Parker's dynamo model taking into account both $\alpha$ and $\omega$ effects operating simultaneously in the two (upper and lower) layers of the solar convective zone (SCZ) and having opposite directions of meridional circulation. The simulations are carried out for both dipole and quadruple magnetic sources with the dynamo parameters specifically selected to provide the curves fitting closely the PCs derived from SBMF variations in cycles 21-23. The simulations are optimised for matching the positions of maximums in latitude, the number of equator crossings and the phase difference between the two dynamo waves operating in the two layers. The dominant pair of PCs present in each cycle is found to be fully asymmetric with respect to the magnetic poles and produced by a magnetic dipole. This pair is found to account for the two main dynamo waves operating
\end{abstract}

between the two magnetic poles. There are also three further pairs of waves unique to each cycle and associated with multiple magnetic sources in the Sun. For the odd cycle 21 the simulated poloidal field fits the observed PCs, only if they are produced by magnetic sources with a quadruple symmetry in both layers, while for the even cycle 22 the fit to the observed PCs is achieved only in the case of quadruple magnetic sources in the upper layer and dipole sources in the inner layer. For the other odd cycle 23 the fit to observation is obtained for the quadruple magnetic sources in the inner layer and the dipole sources in the upper layer. The magnitudes of dynamo numbers $D$ defining the conditions (depth and latitude) of a magnetic flux formation and the numbers $N$ of zeros (equator crossings by the waves) are found to increase and the meridional circulation speed to decrease with a cycle number increase $(D=-700, N=3$ for cycle 21 and $D=-10^{4}, N=9$ for cycle 23). The phase delays between the waves in each unique pairs are also found to increase with the cycle number from $\sim 9^{\circ}$ in cycle 21 to $\sim 13^{\circ}$ in cycle 23 .

Keywords. Solar physics, astrophysics, and astronomy (magnetic fields)

\section{Introduction}

A solar cycle of magnetic activity is manifested with the appearance of sunspots on the surface of the Sun having their number and leading magnetic polarity change with a period of $22 \mathrm{yr}$. These cycles of magnetic activity are associated with the action of solar dynamo mechanisms combining the 
effects of magnetic field shear and stretching caused by the differential rotation ( $\omega$-effect) and twisting of magnetic flux loops allowing them to appear on the surface ( $\alpha$-effect). Such representation provides the solution of dynamo equations in a form of oscillating waves of: (a) the poloidal (background) magnetic field travelling from pole to pole in the whole Sun and (b) the toroidal (sunspot) magnetic fields (dynamowaves) appearing from the middle latitudes to the equator.

The mechanism of such dynamo action was proposed in the fundamental work by Parker (1955). A toroidal magnetic field is produced from the poloidal field by the action of differential rotation. The inverse process of transforming toroidal magnetic field into poloidal field occurs due to the breaking of mirror symmetry by the convection in the rotating body. The action of the Coriolis force on expanding, rising (compressed, sinking) vortices results in a predominance of right-handed vortices in the Northern Hemisphere and left-handed vortices in the Southern Hemisphere. After averaging over velocity pulsations, the electromotive force produced by Faraday electromagnetic induction acquires a component $\alpha B$ parallel to the mean magnetic field $B$. This is the component that closes the self-excitation loop in the Parker's dynamo. This mechanism for generating magnetic fields is the one called " $\alpha$ - $\omega$ dynamo".

In Parker (1955) it is assumed that generation of the dynamo process occurs in a single spherical shell, where twisting of the magnetic field lines ( $\alpha$-effect) and magnetic field line stretching and wrapping around different parts of the Sun owing to its differential rotation ( $\omega$-effect) are acting together. However, it is more than likely that the location of the $\alpha$-effect can be displaced from that of the shear. Moreover, according to the solar rotation curve derived from the helioseismological observations, at least, in some regions of the convective zone the latitudinal shear dominates over the radial shear.

In addition, a meridional circulation is expected to include at least two opposing contributions: one is a flow, which transports solar plasma, say, from the equator to the pole near the surface, and the other is a deeper counter-flow, which returns the plasma to the equatorial region that makes the mass distribution stationary. The above options associate the dynamo generators with different radial layers and consequently, there is a need to include the radial variable into the dynamo-governing equations. Analysis of such a model is much more complex than a one-dimensional example. In order to simplify the problem, Parker (1993) suggested an effective way to describe an interface dynamo with dynamo generators distributed in two radial layers, using for each layer the equations that are very similar to those of the Parker (1955) dynamo.

On the other hand, Babcock (1961) suggested the model of active region eruption and decay with the meridional circulation which is supposed to be a cause of the dynamowave motion. The model was later mathematically defined and analysed by Leighton (1969). A significant distinction of this model from Parker's model was the obligatory presence of the meridional circulation. This model was subsequently developed by Wang et al. (1991), followed by Choudhuri et al. (1995) and Durney (1995) who built flux transport models with the meridional flows in the convective zone.

Given the complexity of the physics in the interior of the Sun, in order to comply with observations, any model should be able to simulate the important features of typical solar cycle (Choudhuri et al., 2004; Dikpati et al., 2004), such as the butterfly diagram, and the phase relation in time between toroidal (sunspot) fields and poloidal (polar) fields. It must do this with the differential rotation and the meridional circulation that are close to that observed in the Sun. In other words, the dynamo models are expected to be calibrated with solar observations.

Recently, a dynamo-based predictive tool was built by converting Babcock-Leighton's calibrated flux-transport dynamo model into a linear system forced from the surface (and not from the convective zone) and applied it to predict the relative sequence of peaks of solar cycles 12 through 24 (Dikpati et al., 2006). By assimilating into the model the surface magnetic-flux data since cycle 12 up to cycle 23 , Dikpati and Gilman (2006) have shown that the first four cycles are required to build its magnetic memory enabling correct prediction of the relative sequence of peaks of cycles 16 through 23. Although this model failed to predict correctly the current very weak solar cycle 24 . In addition, there is an alternate class of flux transport dynamos, where the flux transport is shared, at least, equally by circulation and turbulent diffusion (Nandy and Choudhuri, 2002; Nandy et al., 2011; Choudhuri et al., 2004; Hotta and Yokoyama, 2010; Munoz-Jaramillo et al., 2009; Miesch, 2012). In fact, the two classes of models yield very different predictions of the solar cycle, and it is now somewhat certain that the models relying on an important role of diffusion (Choudhuri et al., 2007) may be closer to the mark (for a weak cycle 24) as concluded from a comparison of these models discussed in the reviews (Jones et al., 2010; Tobias et al., 2011).

Munoz-Jaramillo et al. (2009) undertook the first steps towards a use of more accurate velocity fields in solar dynamo models derived from helioseismic observations for constructing the differential rotation and meridional circulation profiles of magnetic field variations. They present kinematic dynamo simulations driven by the direct helioseismic measurements for the rotation and four plausible profiles for the internal meridional circulation matching the helioseismicallyinferred near-surface depth-dependence. They showed that, in the induction of the toroidal field, the latitudinal shear in the rotation in the bulk of the solar convection zone plays a more important role than either the tachocline or surface radial shears. The speed of the equatorward counterflow in the meridional circulation right at the base of the solar convection zone is found primarily to determine the dynamo cycle period, and not the depth into the radiative interior where it penetrates. 
Nandy et al. (2011) performed kinematic dynamo simulations based on the sunspot numbers in the past $200 \mathrm{yr}$, which demonstrate that the fast meridional flows in the early half phase of solar cycle, followed by a slower flow in the later half phase of the cycle, reproduces the main characteristics of the minimum following the sunspot cycle 23: a large number of spotless days and a relatively weak polar field. The results, therefore, provide a good link between the solar internal dynamics and the atypical values of the heliospheric forcing parameters associated with sunspots, solar radiation, radio flux, cosmic rays, and other characteristics of the solar activity.

In all the above dynamo models it was assumed that there is not a long-term difference in the average levels of the solar activity in the Northern and Southern Hemispheres based on the findings by White and Trotter (1977), who analysed the strongly averaged sunspot data since 1874 . However, later it turned out that for every cycle there are essential differences between the activities in each hemisphere, not only for sunspots (Verma, 1993; Oliver and Ballester, 1994; Temmer et al., 2002; Zharkov and Zharkova, 2006; Zharkov et al., 2008) but also for solar flares (Garcia, 1990; Joshi and Joshi, 2004; Zharkov and Zharkova, 2011), the solar wind (Mursula and Zieger, 2001; Bzowski et al., 2003), long-lived solar filaments (Duchlev, 2001), solar and heliospheric magnetic fields (Bravo and Gonzeles-Esparza, 2000), cosmic-ray propagation (Simpson et al., 1996), and geomagnetic activity (Murayama and Nosaka, 1991). Despite strong North-South Hemisphere asymmetries in many appearances of the solar activity, there is still not a dynamo theory, which can adequately account for this asymmetry.

Even more definitive information was recently obtained with the PCA about the meridional motion of the waves of the magnetic field as seen on the surface with the Wilcox Solar Observatory low resolution whole disk magnetic data (Zharkova and Zharkov, 2010; Zharkova et al., 2012) for cycles 21,22 , and 23 . The authors identified the two main periodical components in latitude of the opposite polarities being common for all three cycles and reflecting two primary waves of SBMF in each hemisphere travelling slightly off-phase and the two secondary (smaller) latitudinal components with appearances varying from cycle to cycle and carrying clear asymmetric features.

This motivated us in the current paper to utilise the previous findings of Zharkov et al. (2008) and Zharkova et al. (2012) about the observed asymmetries in latitudinal distributions of the solar background magnetic fluxes in the first attempt to interpret these results with the simplified dynamo model based on Parker's $(1955,1993)$ models updated with meridional circulation. This approach was selected because a simple model, in which there are only several free parameters, allows one to find more clear mechanisms for physical interpretation of the observational results that can be later used in more realistic numerical models.
The summary of observations is presented in Sect. 2, the governing model equations for simulation of dynamo waves of the poloidal magnetic field are discussed in Sect. 3, the results of the simulation of two waves of poloidal magnetic field for each of cycles 21-23 are presented in Sect. 4 with a general discussion and conclusions drawn in Sect. 5.

\section{Summary of observations}

For a comparison of the patterns observed in the solar magnetic field with predictions by the dynamo model let us use full disk SBMF obtained from the low resolution photospheric magnetic field measurements by the Wilcox Solar Observatory (WSO) (Hoeksema, 1984) in the period between Carrington rotation 1642 and 2036 that covers the past 3 cycles (21-23). In all cycles we consider the WSO SBMF (excluding the sunspots) averaged over longitudes within the $30 \mu$-hemispheres (thin layers parallel to the equator) equally divided between hemispheres $15^{\prime} \times 15^{\prime}$ with respect to the heliographic latitude sines (from $75.2^{\circ} \mathrm{N}$ to $75.2^{\circ} \mathrm{S}$ ) as described by Zharkov et al. (2008).

Let us keep in mind though that SBMF, in fact, is related to a poloidal magnetic field, and it can come from different sources of the dynamo action, possibly, located at different depths. Although, it is not possible to measure directly a precise magnetic field depth distribution in the solar interior, besides some recent inversions of Doppler velocities by means of helioseismology (Schou et al., 1998). Instead, one can substitute these depth distributions with some model simulations by using solar dynamo models, in an attempt to arrive at the same surface distributions which are observed.

For this purpose, we intend to use Parker's two-layer dynamo model, discussed in Sect. 3, which considers the poloidal magnetic field located deeply in the convection zone and directly responsible for solar cycle. Since a direct observation of the poloidal magnetic field in the solar interior is not yet possible, let us consider as its tracer (at least, before finding a better one) the magnetic field on the surface (the photosphere), or SBMF, from which we exclude the magnetic field of sunspots often assumed to be associated with toroidal magnetic field.

PCA of the latitudinal variations of SBMF in all three cycles revealed significant variations between the components with the opposite polarities for different cycles (Zharkova and Zharkov, 2010; Zharkova et al., 2012). Here we extract from Zharkova and Zharkov (2010) the single plot shown in Fig. 1 presenting variations of the magnitude of SBMF versus a latitude. The plot represents variations of the eight PCs derived from PCA: the two components with the largest eigenvalue obtained for the whole dataset of all 3 cycles (two upper curves) and 6 PCs with smaller eigenvalues, supposedly, defining the waves in each cycle separately (the second set of two curves - for the cycles 21, the 3rd set - for cycle 22 and the 4 th, bottom one - for cycle 23). Different 


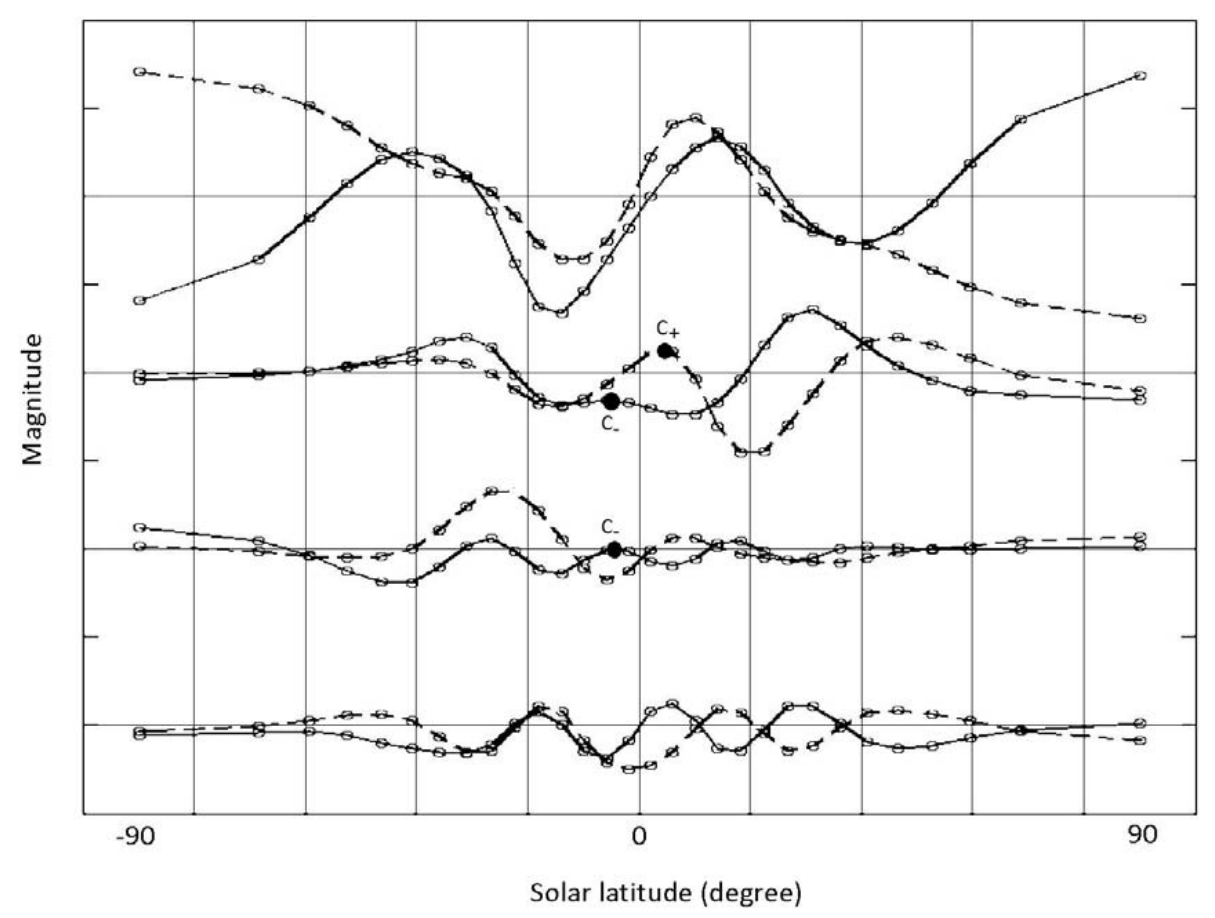

Fig. 1. The latitudinal SBMF distributions in the total set of cycles 21-23 (upper plot), in the separate sets for the cycle 21 , cycle 22 and cycle 23 (from the top to the bottom, respectively). Different components in each pair are shown by the solid and dashed lines. The points $\mathrm{C}_{-}$and $\mathrm{C}_{+}$demonstrate the centres of symmetry of the curves describing the SBMF waves, which roles are discussed in Sects. 4.4 and 4.5 .

components in each pair show the solid and dashed lines. The meaning of the points $\mathrm{C}_{-}$and $\mathrm{C}_{+}$will be explained below in Sects. 4.4 and 4.5 .

In Fig. 1, the horizontal lines correspond to the zero amplitude level for each pair of magnetic field components with positive signs above and negative below. Thus, in each cycle the amplitude of the field repeatedly changes sign along the meridian. For the exact numbers on the $y$ axis corresponding to the curves found for each cycle we refer to Fig. 4 in Zharkova et al. (2012), for example, the maximum of the magnetic field amplitude is approximately 1500 normalised PCs for 21-23 cycles, 1000 - in cycle 21,700 - in cycle 22 , 500 - in cycle 23.

However, since we do not intend to interpret exactly each point of the curves but only their main features, here we use the more schematic Fig. 3 from Zharkova et al. (2012), which represents in one plot the main features for all three cycles (the upper two curves) and for each cycle separately (the further three pairs of curves). We will produce simulations, which the proposed simple model can account for, providing a "face-fit" to the PCs derived from the full disk magnetic field observations on the solar surface, which matches the three basic criteria: the amplitudes of the two waves in both hemispheres, the number of equator-crossings (or zeros) and the phase shifts between the two waves. We will also show that this simple fit allows us to derive some interesting properties of the dynamo processes in different cycles.
By examining the plots in Fig. 1 one can see that the eigenfunctions of the two SBMF components presented in the top plots obtained for the data including all three cycles, resemble rather closely the anti-symmetric waves (towards the equator, e.g. when one wave has minimum the other has maximum and vice versa), or symmetric ones (with respect to the magnetic poles if only an absolute value of magnetic field is considered), which are produced by a simple dipole source. They contain the two principal components of the waves, which are travelling off phase with some time delay between them.

Contrary to the anti-symmetric (with respect to the equator) plots obtained for the two largest eigenvalues, the other three pairs of curves corresponding to the individual cycles show rather different phase shifts and very mixed types of symmetry allowing us to suggest that they are not caused by simple dipole sources but by the quadruple ones. A possibility of excitation of the quadrupole solar magnetic poloidal field mode by the $\alpha-\omega$ dynamo mechanism at the growth phase of activity cycle 23 was first suggested by Krivodubskii (2001, 2005). Using this suggestion and the simple "viewanalysis" of the observed patterns encouraged us to simulate the dynamo waves for dipole and quadrupole components and their mixtures, in order to reproduce the curves in Fig. 1, which are discussed below in Sect. 3. 


\section{Dynamo simulations with meridional circulation}

PCA allowed to distinguish in the observations of SBMF the existence of two waves supposedly assigned to the poloidal magnetic field. Taking into account such the results derived from these observations, let us assume that each wave has its own source. This allows us to use the simplest dynamo model for two layers, which includes the meridional circulation and is, in fact, a one-dimensional dynamo occurring in each thin layer, taking into account advection. This division is supported by the previous dynamo simulations (Ballegooijen and Choudhuri, 1988; Dikpati and Choudhuri, 1995; Munoz-Jaramillo et al., 2011) showing that the diffusivity profiles in the solar interior is a step-like function that can be interpreted in the first approach as two separate layers.

One can see that the latitudinal SBMF distributions shown in Fig. 1 have quite a different structure in different latitudes from the South to the North. To accommodate these differences, one can assume that each of these pairs plotted in Fig. 1 are the result of two different sources of the dynamo, which may be located in different layers, as assumed in the models discussed below.

\subsection{Parker's two-layer dynamo model}

Parker's dynamo equations in the two layers are a development of his model in a single layer by assuming that different dynamo mechanisms operate at different layers. The equations describing the Parker's dynamo can be obtained from the full system of mean-field electro-dynamic equations (Krause and Radler, 1980) by assuming that the waves of solar magnetic field propagate in a thin spherical shell (for example, in the inversion layer). Braginskii (1964; see also Moffatt, 1978) noted that the similar equations can also be derived for a thin cylindrical shell and that the constant meridional circulation can be included into these equations.

The equations are derived by Parker (1993) in such a way that the magnetic field is averaged radially within a certain spherical shell, where the dynamo operates, and terms describing curvature effects near the poles are neglected. Largescale magnetic field has form:

$\mathbf{B}=B(\theta, t) \mathbf{e}_{\phi}+\nabla \times\left(A(\theta, t) \mathbf{e}_{\phi}\right)$.

Here $\phi$ is azimuthal angle, $B(\theta, t) \mathbf{e}_{\phi}$ is the toroidal component, $\nabla \times\left(A(\theta, t) \mathbf{e}_{\phi}\right)$ is the poloidal component. We assume that the magnetic field $B$ and vector potential $A$ are assigned to one layer, while $b$ and $a$ are related to the other layer. Parker's equations for the two layers describing a dynamo with the $\alpha$-effect dominating in one radial layer and the shear of differential rotation ( $\omega$-effect) dominating in the other one are written as follows:

$$
\begin{aligned}
& \frac{\partial B}{\partial t}=\frac{\eta}{n} \Delta B, \quad \frac{\partial A}{\partial t}=\alpha B+\frac{\eta}{n} \Delta A, \\
& \frac{\partial b}{\partial t}=-D \cos \theta \frac{\partial a}{\partial \theta}+\Delta b, \quad \frac{\partial a}{\partial t}=\Delta a .
\end{aligned}
$$

In Parker (1993) it is assumed that the diffusion process has a different intensity in each layer, so $\eta, n$ are the diffusion coefficients in the first and second layers. This assumption was confirmed by the recent kinetic simulations (Munoz-Jaramillo et al., 2011) showing that the simulated diffusivity is a two-step function confirming a presence of two layers with different diffusivity in each. The diffusivity is defined by Eq. (1) in Munoz-Jaramillo et al. (2011) as $\frac{\tau}{3}\left\langle v^{2}\right\rangle$, where $\tau$ is the eddy correlation time and the term with $v$ corresponding to the averaged turbulent squared velocity. $B(r, \theta, t), b(r, \theta, t)$ are the corresponding toroidal magnetic fields in these layers, $A(r, \theta, t), a(r, \theta, t)$ are the vector potentials from which the poloidal magnetic field is derived in each layer.

In the Eqs. (1) and (2) the first terms define change of a magnetic field with time, the terms with $\Delta$ describe diffusion of the magnetic field. Curvature effects are omitted in the diffusion terms (the second terms in the equations for $B$ and $a$ and the third terms for $A$ and $b$ ), so $\Delta=\frac{\partial^{2}}{\partial \theta^{2}}+\frac{\partial^{2}}{\partial r^{2}}$. Parker assumed the differential rotation to dominate in one layer and to vanish in the other, and, conversely, the $\alpha$-effect to prevail in the second layer and to vanish in the first. So action of the differential rotation, which is described by term $\cos \theta \frac{\partial a}{\partial \theta}$, is added to the first Eq. (2), for $b$, and the action of $\alpha$-effect, which is described by the term $\alpha B$, is added to the second Eq. (1), for $A$.

The variables in Eqs. (1)-(2) are normalised by the standard procedure, which is described in Zeldovich et al. (1983) and Krivodubskii (1984), in which $t$ is a diffusion time measuring the time of a magnetic field penetration through the layer where the dynamo works. Since we assume that the turbulent diffusion coefficients are constants in both layers, it is possible to normalise dynamo equations by using one of these coefficients. The diffusion time is proportional to $\frac{\tilde{t} n}{h^{2}}$ where $\tilde{t}$ is the time in real units (for example, in years), $n$ - the turbulent diffusivity coefficient in a layer, $h-\mathrm{a}$ semithickness of the layer. Amplitudes of the $\alpha$-effect and of the angular velocity gradient are combined with the convective diffusivity into a single dimensionless dynamo number $D$ defined as $D=R_{\alpha} R_{\omega}$ and characterising the magnitudes of $R_{\alpha}$ accounting for the contribution of $\alpha$-effect and $R_{\omega}$ - for the differential rotation in the generation of such the dynamo.

Note that $R_{\alpha} \equiv \frac{R \alpha_{0}^{*}}{n}$ is the special magnetic Reynolds number of the $\alpha$-effect where $R$ is solar radius, $\alpha_{0}^{*}$ is the maximum amplitude of the $\alpha$, which is the parameter of the mean helicity of turbulent pulsation. $R_{\omega} \equiv \frac{R^{2}}{n} \frac{1}{r} \frac{\partial \omega}{\partial r}$ is the special magnetic Reynolds number of the differential rotation. Here $\frac{\partial \omega}{\partial r}$ is the radial gradient of the angular velocity, $r$ is the radial distance. In the one-dimensional case $\frac{1}{r} \frac{\partial \omega}{\partial r}$ is the constant (Zeldovich et al., 1983). 


\subsection{Modified dynamo model with meridional circulation}

Popovaa et al. (2010) expanded this model by adding the meridional flows which are described by terms with $V$ and $v$ for each layer, respectively. This results in modified dimensionless equations in the following form:

$$
\begin{aligned}
& \frac{\partial B}{\partial t}+\frac{\partial(V B)}{\partial \theta}=\beta \Delta B, \quad \frac{\partial A}{\partial t}+V \frac{\partial A}{\partial \theta}=\alpha B+\beta \Delta A \\
& \frac{\partial b}{\partial t}+\frac{\partial(v b)}{\partial \theta}=-D \cos \theta \frac{\partial a}{\partial \theta}+\Delta b, \quad \frac{\partial a}{\partial t}+v \frac{\partial a}{\partial \theta}=\Delta a .
\end{aligned}
$$

Here $A, B, a, b$, and $D$ have the same meaning as in Eqs. (1)(2), $\theta$ is the latitude measured from the equator, $\beta=\eta / n$ is ratio of coefficients of turbulent diffusion. The multiplier $\cos (\theta)$ corresponds to the polar shortening of the lines of latitude. If $D$ is large enough, then the solutions of these equations are growing waves, i.e. the effect of dynamo appears. The generation threshold corresponds to the condition under which the generation effects (amplitude of the $\alpha$-effect and differential rotation) are small, so that they are only able to compensate the dissipation losses.

The $V$ in Eq. (3) and $v$ in Eq. (4) represent the meridional circulation defined as velocity of the matter, where waves of the magnetic field propagate. Its structure in each layer is not exactly known, so for simplicity we assume that both $V=$ const and $v=$ const (in the model units). Further, let us assign the meridional circulation with a positive sign if the flows are directed from the equator to the poles and with a negative sign in the opposite case. We accept that in the equations above $\alpha(-\theta)=-\alpha(\theta)$ and the magnetic field is assumed to have a dipolar or quadruple structure.

In the paper Popova et al. (2010) it was suggested that the $\alpha$-effect and differential rotation can act in both layers and the $\alpha$-effect can have a different latitudinal profiles $\alpha_{1}$ and $\alpha_{2}$. Then the equations can be re-written as follows:

$$
\begin{aligned}
& \frac{\partial B}{\partial t}=-D \cos \theta \frac{\partial A}{\partial \theta}+\beta \Delta B, \quad \frac{\partial A}{\partial t}=\alpha_{1} B+\beta \Delta A, \\
& \frac{\partial b}{\partial t}=-D \cos \theta \frac{\partial a}{\partial \theta}+\Delta b, \quad \frac{\partial a}{\partial t}=\alpha_{2} b+\Delta a .
\end{aligned}
$$

This will modify the model described in Eqs. (3)-(4) to the following:

$$
\begin{aligned}
& \frac{\partial B}{\partial t}+\frac{\partial(V B)}{\partial \theta}=-D \cos \theta \frac{\partial A}{\partial \theta}+\beta \Delta B, \\
& \frac{\partial A}{\partial t}+V \frac{\partial A}{\partial \theta}=\alpha_{1} B+\beta \Delta A \\
& \frac{\partial b}{\partial t}+\frac{\partial(v b)}{\partial \theta}=-D \cos \theta \frac{\partial a}{\partial \theta}+\Delta b, \\
& \frac{\partial a}{\partial t}+v \frac{\partial a}{\partial \theta}=\alpha_{2} b+\Delta a .
\end{aligned}
$$

Initially, Parker's dynamo model (Eqs. 1 and 2) had in one layer only a source responsible for the differential rotation and in the other layer - another source responsible for the $\alpha$-effect (described by the parameters $D \cos \theta \frac{\partial a}{\partial \theta}$ and $\alpha B$ as indicated in Eqs. 1 and 2). Hence, in this model, it was necessary to impose such boundary conditions linking these two layers and, thus, inducing the action of a cyclic dynamo associated with $\alpha$ and $\omega$ effects. For the sake of clarity, let us prescribe $r=0$ for the radial boundary between the two layers, and define there the following boundary conditions:

$b=B, \quad a=A, \quad \frac{\partial b}{\partial r}=\beta \frac{\partial B}{\partial r}, \quad \frac{\partial a}{\partial r}=\frac{\partial A}{\partial r}$.

Since the lengths of the latitude net decrease toward the pole, we assume that the material involved in the circulation gradually moves into the counterflow region, while the magnetic field remains in the generation region.

Thus, by assuming that $\alpha$-effect and differential rotation can act in each layer and have different amplitudes one can obtain the following set of equations describing the two layer dynamo model:

$$
\begin{aligned}
& \frac{\partial B}{\partial t}+\frac{\partial(V B)}{\partial \theta}=-D_{1} \cos \theta \frac{\partial A}{\partial \theta}+\beta \Delta B, \\
& \frac{\partial A}{\partial t}+V \frac{\partial A}{\partial \theta}=\alpha_{1} B+\beta \Delta A, \\
& \frac{\partial b}{\partial t}+\frac{\partial(v b)}{\partial \theta}=-D_{2} \cos \theta \frac{\partial a}{\partial \theta}+\Delta b, \\
& \frac{\partial a}{\partial t}+v \frac{\partial a}{\partial \theta}=\alpha_{2} b+\Delta a .
\end{aligned}
$$

Here the differential rotation is described by $D_{1} \cos \theta$ and the alpha-effect is described by $\alpha(\theta)_{1} B$ in the first layer and by $D_{2} \cos \theta$ and $\alpha(\theta)_{2} B$ respectively in the second layer. One can make a reasonable assumption that the intensity of the dynamo may be different in each layer. Thus, we consider a dependence of magnetic field only on latitude, bearing in mind that the information about the depth contained though consideration of different layers which have different values of amplitude of the $\alpha$-effect, differential rotation and meridional circulation.

It was shown earlier (Popova et al., 2010; Popova, 2011) that the solutions of Parker's equations for the toroidal and poloidal magnetic field (either without or with meridional circulation) can have a form of travelling waves, which propagate from the poles to the equator. Since in our problem all the terms (alpha-effect and differential rotation) for generating a magnetic field are present in each layer, we get a solution in the form of two travelling waves, similar to those observed in Zharkova and Zharkov (2010); Zharkova et al. (2012). Note that the meridional circulation has the opposite direction in these layers moving from the equator to the poles and in the deep interior from the pole to the equator 
as suggested by Hathaway (2005) and supported by many authors (Munoz-Jaramillo et al., 2009, 2011, and others). Between the layers there is a region where the meridional flows are practically zero, and it is the boundary of the layers separation.

In the first approach, since we are aiming to reproduce fairly basic features of the PC curves, the details of the exact interaction between the layers are neglected. This approach is validated by a comparison with kinetic simulations of the dynamo models taking into account the meridional circulation as derived from the helioseimology measurements (Munoz-Jaramillo et al., 2009), which showed the separation of meridional flows and the appearance of the region between them with zero flow. This region with zero flow, in fact, can be considered as the boundary between the two layers with meridional circulation, and this boundary can be accepted as non-interactive one as proposed in the current study.

The proposed model was tested with the numerical simulations including the interaction between the layers, which showed that this interaction does not fundamentally change the results for magnetic fields. In fact, for certain values of the coupling coefficients of the dynamo equations with interacting layers we obtained the theoretical results which are similar to the results of a simple model with the noninteracting layers. Hence, we concluded that in the case with the interacting layer between the two layers the influence of dynamo numbers and meridional circulation on the poloidal field is rather similar to that in the case with the two noninteracting layers. Therefore, as the first approach, we can safely investigate the problem with the two non-interacting layers, which has a smaller number of free parameters.

Evidently, adding the boundary conditions between the layers in the model allows us to establish a sufficiently strong connection between the magnetic fields from different layers for the two layer interior medium. However, in the models Eqs. (5), (6), (7)-(10), and (12)-(15) the independent pairs of sources of magnetic fields ( $\alpha$-effect and differential rotation) are suggested to be present in each layer that makes these layers virtually independent for generating different kind of waves.

Since up to-date observations do not allow us to measure exactly how the magnetic sources of the dynamo are connected inside the solar interior, so it is possible to assume that they can work independently in each layer as it was previously suggested, for example, by Benevolenskaya (2000) for modelling the combined 22 yr quasi-biennial cycle. Similarly, here we use a simplified 1-D model with the two layers located in different depths of the interior defining the depth only be these two layers. Then the dynamo systems for these two layers are considered to be independent and, thus, do not need to be connected by any boundary conditions.

\subsection{The final form of the Governing equations}

For simplicity, in the proposed model we assume the same latitudinal profiles of the $\alpha$-effect in each layer, which are equal $\sin 2 \theta$. At the same time, the different dynamo numbers $D_{1}$ and $D_{2}$ and different magnitudes of the meridional circulation $V$ and $v$ are also assumed for the first and second layers, respectively. Thus, one obtains:

$$
\begin{aligned}
& \frac{\partial B}{\partial t}+\frac{\partial(V B)}{\partial \theta}=-D_{1} \cos \theta \frac{\partial A}{\partial \theta}+\beta \frac{\partial^{2} B}{\partial \theta^{2}}, \\
& \frac{\partial A}{\partial t}+V \frac{\partial A}{\partial \theta}=\alpha B+\beta \frac{\partial^{2} A}{\partial \theta^{2}}, \\
& \frac{\partial b}{\partial t}+\frac{\partial(v b)}{\partial \theta}=-D_{2} \cos \theta \frac{\partial a}{\partial \theta}+\frac{\partial^{2} b}{\partial \theta^{2}}, \\
& \frac{\partial a}{\partial t}+v \frac{\partial a}{\partial \theta}=\alpha b+\frac{\partial^{2} a}{\partial \theta^{2}} .
\end{aligned}
$$

Note that the poloidal $\left(B_{\text {poloidal }}=-\frac{\partial A}{\partial \theta}\right)$ and toroidal components of magnetic field may be even functions (for a quadruple structure of magnetic sources) or odd functions (for a dipolar structure). In our model we also assume that the magnitude of meridional circulation as well as the dynamo number $D$ may be different not only in different layers, but also in different hemispheres.

The simplest dynamo model for a layer with a meridional circulation was previously described analytically by using the asymptotic method and then numerically (Popova et al., 2008; Popova and Sokoloff, 2008; Popova et al., 2010) by using WKB (Wentzel-Kramers-Brillouin) technique. As a result, the meridional circulation was shown to regulate the dynamo wave period and, thus, a duration of the activity cycle. Despite Parker's approximation not describing in sufficient detail the polar and equatorial regions, the solar curvature effects cannot dramatically change the simulation result in the main latitudes of the solar activity excluding the poles (Popova, 2010). This means that the plane model can be used as a viable first approximation without large errors in the results.

In the current paper we will simulate a poloidal magnetic field and vector potential for each layer separately, following the assumption made in Sect. 3.2 that the sources of the dynamo in each layer are independent and do not interact. In order to derive the latitudinal distribution for the poloidal magnetic field components, we will use the $Q R$ algorithm, e.g. performing the $Q R$ decomposition by writing the matrix as a product of an orthogonal matrix $\mathbf{Q}$ and an upper triangular matrix $\mathbf{R}$. This algorithm allows us to calculate the eigenvalues and eigenvectors of the matrix defining the magnetic field latitudinal distribution (Ruzmaikin et al., 1980), that is particularly valid for the interpretation of the observational principal components (PCs) derived utilising a similar approach. 


\section{Simulations of two dynamo waves}

\subsection{General comments}

Let us first note that in the proposed dynamo model the following symmetries are accepted across the hemispheres by the sign of poloidal and toroidal magnetic fields: $B(-\theta)=-B(\theta)$ for a dipolar structure and $B(-\theta)=B(\theta)$ for a quadruple structure. This symmetry does not mean the equality of magnetic field magnitudes in each hemisphere, which can be essentially different in the opposite hemispheres as deduced from the observed photospheric magnetic fields (Zharkov et al., 2008; Zharkova and Zharkov, 2010; Zharkova et al., 2012).

This means that the turbulent diffusion, the amplitudes $R_{\alpha}$ of the $\alpha$-effect and $R_{\omega}$ of the differential rotation can be different in the two layers of the SCZ accepted in the updated Parker's dynamo model described above. Since the product of these parameters defines the dynamo number $\left(D=R_{\alpha} R_{\omega}\right)$, we will use the different dynamo numbers $D$ in different layers and in the opposite hemispheres, varying which one can obtain different poloidal magnetic field parity (dipolequadruple) and distributions in latitude that, in turn, can fit those curves derived with PCA from the SBMF (see Fig. 1).

Second, let us assume that the meridional circulation keeps the mass distribution stationary during the dynamo action and, thus, it has to have the opposite signs in the two layers accepted in the model. In other words, one can reasonably suggest that in the top layer located near the surface, the flow transports the solar plasma, say, from the equator to the pole (Hathaway, 2005) and the deeper counter-flow in the other layer returns the plasma to the equatorial region.

Given the fact that PCA derived the pairs of waves shown in Fig. 1, as for the whole set of three cycles, so for each cycle separately, it is also reasonable to assume that each wave in the pair is produced by its own source. Then, we can suggest that one wave is generated in the upper layer of the solar convection zone and another wave coming from its bottom.

And the third characteristic feature in Fig. 1 is the well pronounced asymmetry between the amplitudes of SBMF in the dominant and trailing hemispheres (North dominates South in cycle 21 and South dominates North in cycle 22), while in cycle 23 the amplitudes of magnetic fields in each hemisphere are nearly comparable. Thus, in order to account for this asymmetry, in our simulations different values of meridional circulation are used for cycles 21 and 22 for each layer and each hemisphere, while keeping it the same in both hemispheres for cycle 23 .

Hence, different latitudinal profiles of the waves derived by PCA lead us to assume that the magnitudes of the differential rotation, the $\alpha$-effect, and the speed of meridional circulation are different as in the two layers of the SCZ, and so in the opposite hemispheres. In addition, in order to keep the plasma flow continuous, the meridional circulation in different layers is accepted to move in the opposite directions. Let us now try to produce the theoretical curves of poloidal magnetic field waves generated in the two-layer dynamo model described above for dipole and quadruple magnetic sources, which reasonably fit the observed SBMF distributions by the selected criteria: ratio of the wave amplitudes in the hemispheres, a number of zeros (or equator crossings) and the phase shifts between the two waves for the same model (cycle).

Based on the characteristic features of the whole set and each cycle discussed above, we will consider first in Sect. 4.2 the two main waves (with clearly visible dipole symmetry) common for all cycles 21-23 and then in Sect. 4.3 we investigate the waves with clearly visible dual dipole and quadruple symmetry as seen in cycle 23 . In cycles 21 and 22 none of the symmetries are clearly visible; hence, we will discuss the problem of generation of poloidal magnetic fields and determining their curves symmetry in Sect. 4.4 for cycle 21 and in Sect. 4.5 for cycle 22 .

\subsection{Simulations of two main waves for the cycles 21-23}

Let us first attempt to fit the two curves from the top plot in Fig. 1 presenting the PCs of the SBMF derived for the two strongest eigenvalues of the data for the whole three cycles. One can see that the two SBMF components have a clear dipolar structure because the amplitudes of these waves are clearly anti-symmetric functions relative to the equator (see discussion in Sect. 2) with a small phase shift between them.

The previous simulation results (Popova et al., 2008; Popova and Sokoloff, 2008; Popova et al., 2010) showed that for a fixed value of the dynamo number the inclusion of meridional flows in the two layer dynamo model produces magnetic field waves with a shift in phase. The latitudinal distributions, or butterfly-diagrams, simulated for a few similar dynamo models (Popova et al., 2008) revealed that in a each hemisphere the meridional circulation directed against propagation of the wave strongly shifts the magnetic field to the poles. Thus, one can reasonably assume that the cause for the phase shift in the models with a fixed dynamo number is the presence of meridional flows; and the differences in the shifts in different curves are associated with the difference of their amplitudes in each layer.

The more detailed analysis (Popova et al., 2008) showed that meridional circulation also changes the growth rate of magnetic field and, consequently, the amplitude of the waves along a latitude seen on the solar surface. In addition, a change of the dynamo number $D$ can strongly affect the amplitude of the waves and a number of zeros (points of the equator crossings) because this parameter describes the intensity of the dynamo effect (Parker, 1955). Namely, an increase of the dynamo number increases the number of maxima and minima (or a number of zeros) in the waves and their amplitudes.

Using these general tendencies governing the generation of dynamo waves, let us simulate with the current dynamo 
model (Eqs. 16-19) the main properties of the two dynamo waves with largest eigenvalues derived with PCA for the whole dataset of cycles 21-23 presented in Fig. 1. From the observations of the two top curves in Fig. 1 we can see a pair of waves with clearly antisymmetric amplitudes relative to the equator. This allows us to assume that the amplitudes of meridional circulation to be equal in both hemispheres. Also because the observed magnetic field waves have simple latitudinal profiles with a relatively small amount of zeros ( 5 for one wave (the solid line) and 3 for the another one (the dashed line), so one can also assume that $D$ required for the generation of these waves is not too big (Popova et al., 2008).

Hence, in each layer we can use the same magnitudes of $D$, the parameter responsible for differential rotation and magnetic field twists, because the structure of these waves does not differ significantly in the opposite hemispheres. Although, in order to obtain the phase shift observed in the principal components of the SBMF, we needed to use different values of the meridional circulation in different layers. The results of the simulations reasonably fitting the observations of the SBMF for the whole dataset of cycles 21-23 are plotted in Fig. 2 for the following model parameters: $D_{1}=-700$ and $V=0.3$ for the curve for the upper layer (the solid line) and $D_{2}=-700$ and $v=-0.1$ for the curve for the inner layer (the dashed line).

It can be noted that the solid and dashed curves in this simulation have well defined anti-symmetry relatively to the equator having one "big" maximum and "small" minimum in the negative latitudes and one "big" minimum and "small" maximum in positive latitudes which are defined by their generation by a dipole source. One can also see that we obtained a maximum of the poloidal magnetic field amplitude to be approximately equal to 0.02 model units (such the dimensionless units are used in many dynamo models (see, for example, Zeldovich et al., 1983)), the phase shift is $\sim 8^{\circ}$ (approximately $1 \mathrm{yr}$ ) and the number of zeros is 5 for each wave. Note that a wave of magnetic field passes $90^{\circ}$ during $11 \mathrm{yr}$. Therefore, $1^{\circ}$ corresponds to about $0.12 \mathrm{yr}$, or a $1.0 \mathrm{yr}$ difference corresponds about to $8.1^{\circ}$ of the phase shift. The amplitude, number of zeros and the phase shift derived from PCA for the all three cycles 21-23 resemble the theoretical results in the middle latitudes where Parker's two layer model is applicable.

Hence, the simulations show that the magnetic waves with the highest eigenvalues in Fig. 1 are produced by the dipole magnetic sources with the opposite polarities present in both inner and outer layers, and these dipoles are those located at the poles. This is a prototype of the standard two dipole dynamo model with the two poles, although, corrected with the meridional circulation. In order to fit the observed top curves, the meridional circulation needs to be slightly faster in the upper layer $(V=0.3)$ compared to the inner one $(v=-0.1)$. This can cause a faster exchange of polarities in the outer layer compared to the inner one and lead to the noticeable phase shift between the waves.

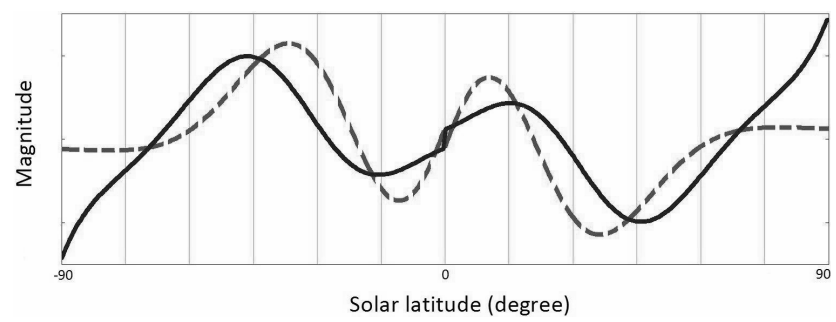

Fig. 2. Simulations for the two wave components of poloidal magnetic field generated in the upper and inner layers (solid line and dashed line, respectively) reproducing reasonably close (within limitations of the simple model used as discussed in Sect. 4.1) the main features of the SBMF top curves in Fig. 1 obtained for cycles 21-23 (for details of comparison see the text). The following model parameters are used: $D_{1}=-700$ and $V=0.3$ for the curve shown by the solid line and $D_{2}=-700$ and $v=-0.1$ for the curve shown by the dashed line.

\subsection{Simulations of two waves for the odd cycle 23}

The next set of curves, which we wish to interpret, is presented at the bottom in the Fig. 1 for the components obtained for the odd cycle 23 . The curve shown by the solid line has a clear anti-symmetric structure relative to the equator (see Sect. 2), thus, it is generated by a dipolar magnetic source. While the curve shown by the dashed line is symmetric relative to the equator, allowing us to assume that it is produced by a quadruple source. This assumption is confirmed by the cross-correlation of the PCA data for cycle 23 presented by Zharkova et al. (2012), their Fig. 6, which revealed a presence of three dipoles for this cycle, or one dipole and one quadruple. Based on this finding, we assume a one dipole to be present in the upper layer and the other two dipoles (located in perpendicular directions and, thus, forming a quadruple) - in the inner layer. As mentioned in Sect. 4.1, for cycle 23 the wave asymmetry between the hemispheres is small and, thus, not considered in this model simulation.

It has to be noted though that the timing of the appearance of the quadruple sources is not defined in PCA, since for the analysis the whole dataset was considered for the duration of each cycle (10-11 yr). This means that the quadruple sources can be, in fact, present only during the short period of a few years in the growth phase of the poloidal magnetic field and disappear at later times as previously reported by Krivodubskij (2005) and Nandy et al. (2011).

The amplitudes for each of these waves of the SBMF have 8 zeros that is about twice more than in the case for the waves derived for all three cycles discussed in Sect. 4.2. Hence, in order to obtain such a wave structure as derived for cycle 23 , one needs to use a larger dynamo-number than in the case for all three cycles. Also the amplitudes of the derived SBMF waves are nearly equal, they have a smaller phase shift (about $5^{\circ}(0.6 \mathrm{yr})$ in the middle latitudes) in the Northern 
Hemisphere and are nearly in the anti-phase (a phase shift is about $\left.14^{\circ}(\sim 1.7 \mathrm{yr})\right)$ in the Southern Hemisphere.

This allows us to suggest that absolute magnitudes of the meridional circulation are equal in both layers while the antiphase of the wave in the Southern Hemisphere noted above can arise from a combination of the dipole and quadruple magnetic sources present in these two layers. We accept the meridional circulation to be equal to 0.2 and $D_{1}=-10^{4}$ for the upper layer (where there is a dipole source) and to -0.2 and $D_{2}=-10^{4}$ for the inner layer (where there is a quadruple source) and plotted the results in Fig. 3.

Since the larger dynamo number is used, the maximum of the simulated poloidal field amplitude is approximately equal to 0.07 model units that is higher than in the pure dipole case used for all three cycles. This increase complies with the previous dynamo simulations showing that an increase of the amplitudes of either differential rotation or alpha-effect leads to an increase in the intensity of the dynamo generation and, hence, to an increase in the amplitude of a magnetic field wave (Popova, 2008).

Also the simulation in Fig. 3 predict the phase shift of $\approx 16^{\circ}(\sim 1.9 \mathrm{yr})$ between the waves generated by different layers for the positive middle latitudes resembling those nearly in anti-phase derived with PCA analysis in Fig. 1 and $\approx 3^{\circ}(\sim 0.4 \mathrm{yr})$ for the negative middle latitudes that resembles the phase shifts at the middle latitudes between the observed bottom curves shown in Fig. 1. The magnitude of each wave has 9 zeros, which number fits rather closely the number of 8 derived from the PCA. Note that near the equator the theoretical quadruple curve is different from the observations, but this happens in the low latitude where our model is not applicable. Hence, one can conclude that under the terms of applicability of the model (middle latitudes), the observed SBMF and theoretical waves for poloidal magnetic field have similar latitudinal profiles for the dipole structure of magnetic sources in the outer layer and the quadruple one in the inner layer.

\subsection{Simulations of two waves for the odd cycle 21}

For the odd cycle 21, the pair of the SBMF waves obtained with PCA is shown in Fig. 1, the second row from the top. Both waves reveal rather symmetric structures towards the equator indicating a quadruple symmetry of the magnetic sources generating these waves. Also the curves have equal numbers of their maxima and minima, that also supports the quadruple symmetry. However, the symmetric structure of the SBMF sign $\left(B_{\mathrm{SBMF}}(-\theta)=B_{\mathrm{SBMF}}(\theta)\right)$ is not kept with respect to the equator but to the other symmetry points shifted from the equator to the North or South. Namely, the curve shown by the solid line has a centre of the quadruple symmetry in the point $\mathrm{C}_{-}$(shifted to the South), and the curve shown by the dashed line has a centre of the quadruple symmetry in the point $\mathrm{C}_{+}$(shifted to the North).

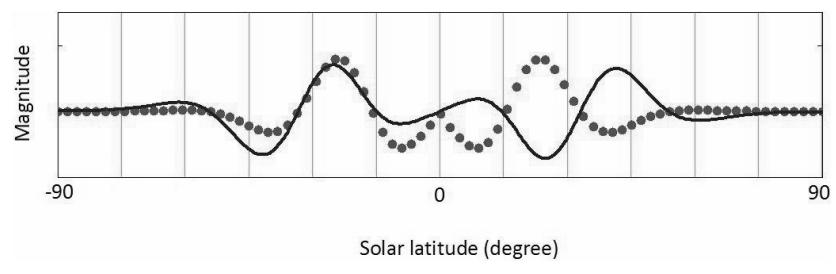

Fig. 3. Simulations of poloidal magnetic field for the dipole (solid line with $D_{1}=-10^{4}, V=0.2$ ) and quadruple (dotted line with $D_{2}=-10^{4}, v=-0.2$ ) magnetic sources reproducing the latitudinal SBMF distributions for cycle 23 .

Unlike the previous two cases discussed in Sects. 4.2 and 4.3 , in which the maxima of the SBMF magnitudes were nearly equal in the opposite hemispheres, in cycle 21 both the waves have larger maxima in the Southern Hemisphere compared to those in the Northern one. Hence, one can assume that for cycle 21 there is some factor, which has a different effect on the generation of poloidal field waves in the opposite hemispheres. A factor, which can change the magnitude of the poloidal field is a meridional circulation speed, which has to be more intense in the Southern Hemisphere compared to the Northern one.

In addition, because the two curves for cycle 21 have rather different shapes, this means that, for their reproduction with the dynamo model, the meridional circulation in different layers has to have different velocities. Since the amplitudes of both the PCA waves with quadruple structures have a smaller number of the local extrema ( 7 versus 9$)$ than in the previous case (for cycle 23), to reproduce them in the dynamo model, one needs to use a smaller dynamo number than in cycle 23 . Therefore, the parameters accepted to simulate the waves described the PCA curves for cycle 21 are the following: for the upper layer $-D_{1}=-700, V=0.8$ (region of the positive latitude), and $V=0.3$ (region of the negative latitude) (dashed line) and for the inner layer $-D_{2}=-500$, $v=-0.1$ (region of the positive latitude), and $v=-0.3$ (region of the negative latitude) (solid line).

The simulation results are plotted in Fig. 4, which show that we managed to obtain the model curves with the structure qualitatively similar to that observed in the PCA curves. Similar to the observations, the amplitudes of the model waves are larger in the Northern Hemisphere than in the Southern Hemisphere; and they also have the same number 7 of the local extrema. The maximum of the SBMF magnitude for these waves is approximately equal to 0.02 model units that is much smaller than in the case for cycle 23 . This occurs because the dynamo numbers used are two orders of magnitude smaller compared to cycle 23 .

The model phase shifts between the two simulated waves at the middle latitudes are $\approx 15^{\circ}(\sim 1.8 \mathrm{yr})$ for the positive latitudes and $\approx 10^{\circ}(\sim 1.2 \mathrm{yr})$ for the negative one. At the same time, the observable phase shift is $\approx 10^{\circ}$ for the positive latitudes and a few degrees for the negative one in the middle 


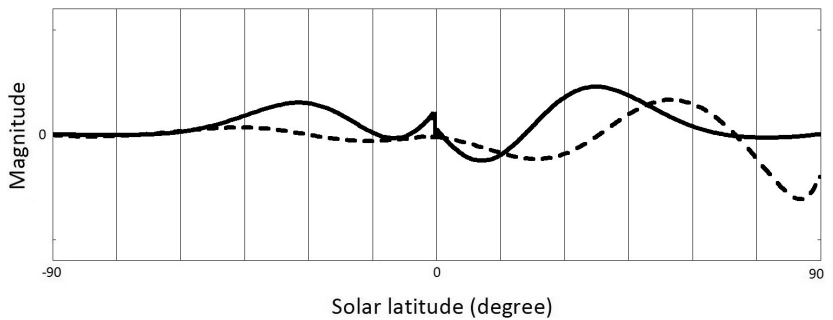

Fig. 4. Simulations for the two quadruple components of the poloidal magnetic field reproducing the latitudinal SBMF distribution for cycle 21: dashed line with the parameters: $D_{1}=-700$, $V=0.8$ (region of positive latitude), and $V=0.3$ (region of negative latitude); solid line with the parameters: $D_{2}=-500, v=-0.1$ (region of positive latitude), and $v=-0.3$ (region of negative latitude).

latitudes. Such a difference in the phase shifts between the model and observations in the Northern Hemisphere can be associated with the fact that the modified Parker's model used in the current simulations is symmetric towards the equator, unlike the observations, and does not take into account a shift of the centre of the symmetry detected in the principal components for this cycle in Fig. 1.

Hence, in the odd cycle 21 there are clear indications of the presence of the two quadruple magnetic sources, one in each layer, required for fitting the observed curves with the Parker's two-layer dynamo model used in the current paper. This is different from the odd cycle 23 where the quadruple structure was required to be only present in the inner layer wave while the outer layer wave was well reproduced by a dipole structure.

Although, we need to remember that the principal components used in this study are found for the whole cycle data, and thus, the timing of the appearance of the quadruple sources is not defined. This means that the quadruple sources can, in fact, only be present during the short period of time of a few years in the growth phase of the poloidal magnetic field as it was previously pointed by Krivodubskij (2005) and Nandy et al. (2011).

\subsection{Simulations of two waves for cycle 22}

Let us now look at the last set of plots for the even cycle 22 presented in Fig. 1 by the third from the top set of curves. Similar to cycle 23 , the amplitude of the wave shown by the dashed line for cycle 22 is almost anti-symmetric relative to the equator that looks like a dipolar structure while the amplitude of the wave shown by the solid line is almost symmetric relative to the point of symmetry $C_{-}$(shifted to the south) that looks like a quadruple structure. In the Northern Hemisphere the wave shown by the dashed line has a larger amplitude in comparison to the other wave shown by the solid line while in the Southern Hemisphere both waves have comparable but smaller amplitudes than in the Northern Hemisphere.

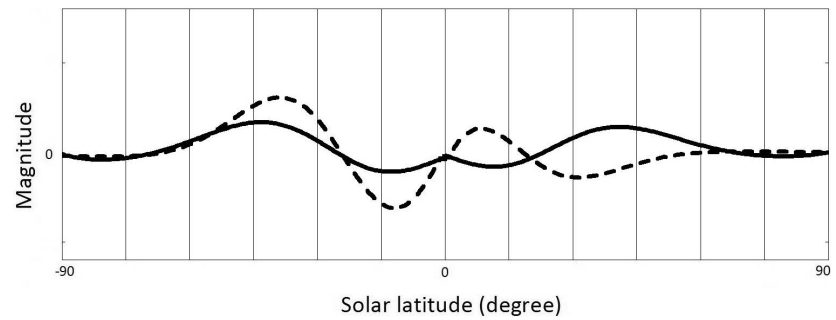

Fig. 5. Simulations for the two components of the poloidal magnetic field reproducing the latitudinal SBMF distribution for cycle 22. The solid line is obtained with $D_{1}=-400, V=0.2$ (region of negative latitude), and $V=0.1$ (region of positive latitude), the dashed line - with $D_{2}=-1000, v=-0.1$ (region of negative latitude), and $v=-0.5$ (region of positive latitude).

By applying the general analysis described in Sect. 4.1 to the curves, we deduce that for fitting the latitudinal distributions of the SBMF with the model, we need to select a larger dynamo number for simulation of the dipolar wave generated in cycle 22 (Fig. 5), in order to provide a larger amplitude of the wave, and to use a different value of the meridional circulation in different hemispheres, in order to provide a difference in magnitude and phase between the wave amplitudes of the two curves.

Thus, the dipolar wave for the inner layer is reproduced by using $D_{1}=-1000, V=-0.5$ (region of the positive latitude), and $V=-0.1$ (region of the negative latitude) (dashed line) and the quadruple wave for the upper layer is reproduced by using $D_{2}=-400, v=0.1$ (region of the positive latitude), and $v=0.2$ (region of the negative latitude) (solid line). The simulation results reproducing the SBMF variations in cycle 22 are plotted in Fig. 5. The simulations show that the SBMF amplitude of the model dipole wave has 6 zeros similar to the observed PCA wave. The amplitude of the model and observed quadruple wave both have 7 zeros. Also the maximum magnitude in the model is $\approx 0.03$. In the Northern Hemisphere the phase shift between the two waves in the model is about $\approx 5^{\circ}(\sim 0.6 \mathrm{yr})$, in the Southern Hemisphere the waves are nearly in the anti-phase that is close to those found from the observations. There is an interesting trend in the simulated waves: in cycle 22 the wave generated by the inner layer has the dipolar symmetry and the wave from the upper layer is still reproduced by the quadruple one, unlike the symmetry of magnetic waves in the odd cycle 21 which were the quadruple ones in both layers. However, this symmetry is opposite to that found in cycle 23 where the dipolar symmetry appears in the upper layer and the quadruple symmetry is in the inner one. Also the centre of symmetry for the wave caused by quadruple sources in cycle 22 is shifted to the South, similarly to cycle 21 .

This indicates that the conditions of dynamo generation have to be changed significantly from cycle 21 to cycle 23 not only in the dynamo numbers and meridional circulation parameters in the layers and in the hemispheres but also in 
Table 1. Model parameters in cycles.

\begin{tabular}{|c|c|c|c|c|}
\hline Cycle & 21 & 22 & 23 & $21-23$ \\
\hline \multicolumn{5}{|c|}{ Upper layer } \\
\hline Dymano number & $-700 \pm 50$ & $-400 \pm 50$ & $-10^{4} \pm 500$ & $-700 \pm 50$ \\
\hline Meridional circulation (region of positive latitude) & $0.8 \pm 0.05$ & $0.1 \pm 0.05$ & $0.2 \pm 0.05$ & $0.3 \pm 0.05$ \\
\hline Meridional circulation (region of negative latitude) & $0.3 \pm 0.05$ & $0.2 \pm 0.05$ & $0.2 \pm 0.05$ & $0.3 \pm 0.05$ \\
\hline \multicolumn{5}{|c|}{ Inner layer } \\
\hline Dymano number & $-500 \pm 50$ & $-1000 \pm 70$ & $-10^{4} \pm 500$ & $-700 \pm 50$ \\
\hline Meridional circulation (region of positive latitude) & $-0.1 \pm 0.05$ & $-0.5 \pm 0.05$ & $-0.2 \pm 0.05$ & $-0.1 \pm 0.05$ \\
\hline Meridional circulation (region of negative latitude) & $-0.3 \pm 0.05$ & $-0.1 \pm 0.05$ & $-0.2 \pm 0.05$ & $-0.1 \pm 0.05$ \\
\hline
\end{tabular}

the numbers and locations of magnetic sources in the solar interior.

\section{Discussion and conclusions}

In the current paper, we made an attempt to reconstruct qualitatively with a simple Parker's model of the two layer dynamo the basic features in the latitudinal distributions of solar background magnetic field (SBMF) (associated with solar poloidal field) in cycles 21-23 derived with the Principal Component Analysis (PCA). The observational curves described in Sect. 2 were presenting big puzzles for their association with the actions of the solar dynamo. For the first time we have interpreted these curves with the two layers Parker's models and showed the conditions, under which the simplest dynamo theory is able to explain these observational results.

It was shown that the PCs (see Fig. 1) derived from SBMF latitudinal structure are likely to reflect different conditions of the dynamo generation in latitude and depth, which can be reasonably reproduced only by using different magnitudes of the dynamo number and of the meridional circulation changing in different hemispheres of the same cycle and also from cycle to cycle. Our goal was to derive the dynamo properties of the pairs of waves of poloidal magnetic field which closely resemble those obtained with PCA in Fig. 1 for the whole set of cycles 21-23 and for each cycle separately.

Based on the general properties of the observations, one can suggest that the observable pairs of SBMF components can be the result of the dynamo action with the intensity and parity being different in depth. This allowed us to replace the two-dimensional model with the two one-dimensional ones, with the amplitude of the dynamo assumed different in depth. In the proposed dynamo model we consider the two layers where the poloidal magnetic field can be generated by the solar dynamo machine: the inner layer located at the bottom of the SCZ and the outer layer located at the very top of the SCZ. The meridional circulation has opposite direction in these layers (Hathaway, 2005). Furthermore, according to the results of Popova (2009), the model meridional circulation (which is about $10^{-1}$ model unit) corresponds $t$ the flow of $10^{-2} \mathrm{grad} /$ day for the range of the obtained dynamo numbers that is quite a reasonable assumption (Hathaway, 2005). Between the layers there is a boundary region where the meridional flows are practically zero, which is the boundary of the layers separation imposing the lack of interference between the layers.

We suggest that in different cycles the amplitudes of the $\alpha$-effect, $R_{\alpha}$, and of the differential rotation, $R_{\omega}$, can be different in these two layers as it was shown earlier (Popova et al., 2010). Since these parameters define the dynamo number $\left(D=R_{\alpha} R_{\omega}\right)$, we used the different dynamo numbers $D$ in the two layers and in the opposite hemispheres, in order to find the best fitting of the simulations to those waves derived with PCA (see Fig. 1). In the Table 1 we present the parameters in our models used for fitting the PC components for each cycle and for the whole dataset with the estimated set of errors reflecting the accuracy, with which changing the model parameters leads to smooth changes of the latitudinal profile of magnetic field.

Strictly speaking, the fit of the theory to the PCs found from the observations is not the fully mathematically-defined fit of each observational point allowing to estimate standard deviations and confidence intervals. It is more like a "facefit", which matches the three basic criteria of the PCs derived from the magnetic field observation on the solar surface: the amplitudes in the opposite hemispheres, the number of equator-crossings and the phase shifts between the two curves. The more advanced dynamo model allowing us a one-to-one fit of the theory to observations is yet to be developed.

However, with the "face-fit" we can roughly estimate the errors of the fit by choosing the limits defining the error bars. If the theoretical waves fit reasonably well the observed curves in all three parameters (ratio of amplitudes in the opposite hemispheres, number of zeros and the phase shift between the curves) as discussed above we consider the fit to be within the error bar limits, while outside these limits the curves do not resemble the observational PCs in any 
of three parameters, or deviate from the PCs in at least two parameters.

The poloidal magnetic field is considered to be generated by the two kinds of magnetic sources, producing the two types of symmetry: the dipolar and quadruple ones. One can naturally assume that the PCA pair of the curves for the all the set derived for 3 cycles are the waves of the poloidal magnetic field produced from the inner and outer layers of the SCZ by the combined action of the two dynamo sources, which can also have different parameters in the opposite hemisphere and different cycles. Perhaps, these variations are caused by the geometry of plasma flows in the convection zone, which can vary from cycle to cycle.

We are fully aware that the model used in the current study is a very simple one, which, nonetheless, allows us to test our understanding of the physical processes responsible for the magnetic field generation during solar activity cycles that can lead to some essential modifications of more sophisticated models, which simulate the solar activity in much more detail (for example, see Dikpati et al., 2006 and review of different models Brandenburg and Subramanian, 2005). However, this simplified approach allows us to derive basic properties of the waves generated in the whole set of cycles 21-23 and to establish how these properties can change in each separate cycle.

The reproduction of the pair of SBMF component with the largest eigenvalues derived for the total set for all cycles 2123 required to model these two waves of the poloidal magnetic field produced by a dipolar structure with a low dynamo number $(-700)$ as indicated in Sect. 4.2. This includes the fit of amplitude ratios in the opposite hemispheres and the numbers of zeros, as well as the phase shift between the observed pair of waves, which is approximately equal $7^{\circ}$ in the middle latitudes and the model shift of about $8^{\circ}$. This pair of waves is considered to emulate the classic dynamo with the dipole magnetic source, which is considered to be responsible for regeneration of the poloidal and toroidal magnetic fields of the Sun (Parker, 1955).

However, the conditions of the magnetic field generation fitting the derived PCs in SBMF are found to change significantly from cycle 21 to cycle 23 including the changes in the dynamo numbers and the meridional circulation parameters in the layers and in the hemispheres as discussed in Sects. 4.3-4.5. The best fits of the simulated poloidal magnetic field waves to PCs shown in Fig. 1 for separate cycles allowed us to establish the following parity rules: for cycle 21 the quadruple magnetic field sources are found located in both layers, for cycle 22 - quadruple sources are located only in the outer layer and the dipole ones in the inner layer and for cycle 23 - quadruple magnetic sources in the inner layer and the dipole magnetic sources in the outer one.

Although, nothing can be said about the timing of the appearance of the quadruple sources in cycles, since for the analysis the whole dataset was considered for the duration of each cycle (10-11 yr) not separating the phases of cycles.
This means that the quadruple sources can be, in fact, present only during the short period of a few years in the growth phase of the poloidal magnetic field and disappear at later times previously reported by Krivodubskij (2005) and Nandy et al. (2011).

The simulations fitting the observed PC curves (see Fig. 1) clearly show that with an increase of the cycle number there is the increase of the dynamo number $D$, which comprises of the amplitudes of the differential rotation and the $\alpha$-effect, and in the number of the poloidal magnetic field zeros. This is reproduced by the variations of the dynamo number from $D=-10^{4}$ for cycle 23 where the $8(9)$ zero numbers are present in PCA (model) to $D=-700$ for cycle 21 where only 3 zeros are present. In addition, for all the three cycles the poloidal magnetic field waves are found to have an increasing phase shift in the negative latitudes approaching nearly an anti-phase in the positive latitudes. In general, a range of the derived dynamo numbers and the meridional circulation is quite realistic for solar physics according results of Popova (2009), Popova et al. (2008), Tuominen et al. (1983), Komm et al. (1993), Zhao and Kosovichev (2004) and Brandenburg and Subramanian (2005).

It has to be noted that, in order to change the parameters of solar cycle (amplitude, period, and phase), alternative approaches can be also used. For example, Munoz-Jaramillo et al. (2011) show by using more sophisticated kinematic dynamo simulations involving the coupling of magnetic quenching with variable turbulent diffusivity allowed the authors to reproduce some realistic parameters of the recent solar cycles (e.g. a prolonged solar minimum of cycle 24). In our study the turbulent diffusivity is included in dynamonumber and can change in combination with the amplitudes of the $\alpha$-effect and differential rotation the profile of the wave in each layer. Thus, such the phenomenon as a difference in the poloidal field amplitude maxima in opposite hemispheres during the cycle must be related to the asymmetry in the value of some physical parameters.

In addition, there is an interesting observation derived from the simulations with meridional circulation. The increase of an amplitude of the meridional circulation In the upper layer leads to an increase of the magnetic field amplitude and decrease of its amplitude in the inner layer. This tendency is also valid for the toroidal field. This finding correlates rather well with the 2-D simulation results by Munoz-Jaramillo et al. (2009) showing that in the shallow area $\left(>0.71 R_{\odot}\right)$ the larger value of the amplitude of the meridional circulation corresponds to the larger amplitude of the magnetic field while the tendency is opposite in the deeper area $\left(<0.64 R_{\odot}\right)$. However, unlike Munoz-Jaramillo et al. (2009), in our models, in order to reproduce the amplitudes of significantly asymmetric PCs derived from the observational data, in each hemisphere different amplitudes of the meridional circulation are used.

Therefore, the first quantitative interpretation of the pairs of magnetic field waves derived with PCA from the 
observations of SBMF observed on the solar surface uncovered very important effects of the conditions required to generate the similar waves from the Parker's two-layer dynamo model. These include different parity rules (dipolequadruple), different dynamo numbers and meridional circulation speeds in the two layers for different cycles and even for different hemispheres within the same cycle. This indicates that the conditions of the magnetic field generation by the dynamo mechanism are likely to be changed significantly from cycle 21 to cycle 23 including the dynamo numbers at different depths (or layers) that was anticipated (Charbonneau, 2005) and the meridional circulation parameters in the layers and in the hemispheres (Hathaway, 1996). These conditions for generation of SBMF, in turn, can significantly affect the generation of the toroidal magnetic field appearing on the solar surface as sunspots, which numbers define the solar activity that will be a scope of a forthcoming paper.

Acknowledgements. The authors acknowledge the financial support from the Russian Fundamental Research Foundation under the grants 12-02-31128, 12-02-00170, 12-02-00884, 10-02-00960, 1402-00284 and the support by the Royal Society Joint International grant, UK, during which the research was initiated. The authors wish to thank the staff of the Wilcox Solar Observatory for supplying their data for a free use by researchers. Also the authors wish to expressed their deepest appreciation to the referees for very constructive and useful comments, from which paper strongly benefited.

Topical Editor M. Balikhin thanks V. Krivodubskij, L. Ofman, and one anonymous referee for their help in evaluating this paper.

\section{References}

Babcock, H. W.: The Topology of the Sun's Magnetic Field and the 22-year Cycle, Astrophys. J., 133, 572-587, doi:10.1086/147060, 1961.

Ballegooijen, A. A. and Choudhuri, A. R.: The possible role of meridional flows in suppressing magnetic buoyancy, Astrophys. J., 333, 965-977, doi:10.1086/166805, 1988.

Benevolenskaya, E. E.: A mechanism of helicity variations on the Sun, Solar Phys., 191, 247-255, doi:10.1023/A:1005211501835, 2000.

Braginskii, S. I.: Kinematic Models of the Earth's Hydromagnetic Dynamo, Zh. Eksp. Teor. Fiz., 48, 2178-2193, 1964.

Brandenburg, A. and Subramanian, K.: Astrophysical magnetic fields and nonlinear dynamo theory, Phys. Rept., 417, 1-209, doi:10.1016/j.physrep.2005.06.005, 2005.

Bravo, S. and Gonzeles-Esparza, J. A.: The north-south asymmetry of the solar and heliospheric magnetic field during activity minima, Geophys. Res. Lett., 27, 847-849, doi:10.1029/1999GL010709, 2000.

Bzowski, M., Makinen, T., Kyrola, E., Summanen, T., and Quemerais, E.: Latitudinal structure and north-south asymmetry of the solar wind from Lyman-alpha remote sensing by SWAN, Astron. Astrophys., 408, 1165-1177, doi:10.1051/00046361:20031022, 2003.
Charbonneau, P.: Dynamo Models of the Solar Cycle, Living Rev. Solar Phys., 2, 2-91, doi:10.12942/lrsp-2010-3, 2005.

Choudhuri, A. R., Schussler, M., and Dikpati, M.: The solar dynamo with meridional circulation, Astron. Astrophys., 303, L29-L32, 1995.

Choudhuri, A. R., Chatterjee, P., and Nandy, D.: Helicity of Solar Active Regions from a Dynamo Model, Astrophys. J. Lett., 615, L57-L60, doi:10.1086/426054, 2004.

Choudhuri, A. R., Chatterjee, P., and Jiang, J.: Predicting Solar Cycle 24 With a Solar Dynamo Model, Phys. Rev. Lett., 98, 131103, doi:10.1103/PhysRevLett.98.131103, 2007.

Dikpati, M. and Choudhuri, A. R.: On the Large-Scale Diffuse Magnetic Field of the Sun, Solar Phys., 161, 9-27, doi:10.1007/BF00732081, 1995.

Dikpati, M. and Gilman, P. A.: Simulating and Predicting Solar Cycles Using a Flux-Transport Dynamo, Astrophys. J., 649, 498514, doi:10.1086/506314, 2006.

Dikpati, M., Toma, G., Gilman, P. A., Arge, C. N., and White, O. R.: Diagnostics of Polar Field Reversal in Solar Cycle 23 Using a Flux Transport Dynamo Model, Astrophys. J., 601, 1136-1151, doi:10.1086/380508, 2004.

Dikpati, M., Toma, G., and Gilman, P. A.: Predicting the strength of solar cycle 24 using a flux-transport dynamo-based tool, Geophys. Res. Lett., 33, L05102, doi:10.1029/2005GL025221, 2006.

Duchlev, P. I.: An Estimation of the Long-Term Variation of a North-South Asymmetry of the Long-Lived Solar Filaments, Solar Phys., 199, 211-215, doi:10.1023/A:1010313817889, 2001.

Durney, B. R.: On a Babcock-Leighton dynamo model with a deepseated generating layer for the toroidal magnetic field, Solar Phys., 160, 213-235, doi:10.1007/BF00732805, 1995.

Garcia, H.: Evidence for solar-cycle evolution of north-south flare asymmetry during cycles 20 and 21, Solar Phys., 127, 185-197, doi:10.1007/BF00158522, 1990.

Hathaway, D. H.: Doppler Measurements of the Sun's Meridional Flow, Astrophys. J., 460, 1027-1033, doi:10.1086/177029, 1996.

Hathaway, D. H.: How Large-scale Flows May Influence Solar Activity?, Conference proceedings ASP series, 346, 19, 2005.

Hoeksema, J. T.: Ph.D. Dissertation, Center for Space Science and Astrophysics, Stanford University, California, 1984.

Hotta, H. and Yokoyama, T.: Importance of Surface Turbulent Diffusivity in the Solar Flux-Transport Dynamo, Astrophys. J., 709, 1009-1017, doi:10.1088/0004-637X/709/2/1009, 2010.

Jones, C. A., Thompson, M. J., and Tobias, S. M.: The solar dynamo, Space Sci. Rev., 152, 591-616, doi:10.1007/s11214-0099579-5, 2010.

Joshi, B. and Joshi, A.: The North-South Asymmetry of Soft X-Ray Flare Index During Solar Cycles 21, 22 and 23, Solar Phys., 219, 343-356, doi:10.1023/B:SOLA.0000022977.95023.a7, 2004.

Komm, R. W., Howard, R. F., and Harwey, J. M.: Meridional Flow of Small Photospheric Magnetic Features, Solar Phys., 147, 207223, doi:10.1007/BF00690713, 1993.

Krause, F. and Radler, K.: Mean Field Magnetohydrodynamics and Dynamo, Akademie, Berlin, 1980.

Krivodubskii, V. N.:Intensity of Sources of Magnetic Fields of the Solar Alpha-Omega Dynamo, Soviet Astron., 61, 540-548, 1984. 
Krivodubskii, V. N.: The structure of the global solar magnetic field excited by the turbulent dynamo mechanism, Astron. Reports, 45, 738-745, doi:10.1134/1.1398923, 2001.

Krivodubskii, V. N.: Turbulent dynamo near tachocline and reconstruction of azimuthal magnetic field in the solar convection zone, Astron. Nachr., 326, 61-74, doi:10.1002/asna.200310340, 2005.

Leighton, R. B.: A Magneto-Kinematic Model of the Solar Cycle, Astropys. J., 156, 1-26, doi:10.1086/149943, 1969.

Miesch, M.: The solar dynamo, Philos. T. Roy. Soc. A, 370, 30493069, doi:10.1098/rsta.2011.0507, 2012.

Moffatt, H. K.: Magnetic Field Generation in Electrically Conducting Fluids, Cambridge University Press, Cambridge, 1978.

Munoz-Jaramillo, A., Nandy, D., and Martens, C. H.: Helioseismic Data Inclusion in Solar Dynamo Models, Astophys. J., 698, 461478, doi:10.1088/0004-637X/698/1/461, 2009.

Munoz-Jaramillo, A., Nandy, D., and Martens, C. H.: Magnetic Quenching of Turbulent Diffusivity: Reconciling Mixing-length Theory Estimates with Kinematic Dynamo Models of the Solar Cycle, Astrophys. J. Lett., 727, L23-L29, doi:10.1088/20418205/727/1/L23, 2011.

Murayama, T. and Nosaka, T.: Long-term variation in the northsouth asymmetry of geomagnetic activity with respect to the solar equatorial plane, Planet. Space Sci., 39, 751-760, doi:10.1016/0032-0633(91)90070-Q, 1991.

Mursula, K. and Zieger, B.: Long-term north-south asymmetry in solar wind speed inferred from geomagnetic activity: A new type of century-scale solar oscillation?, Geophys. Res. Lett., 28, 9598, doi:10.1029/2000GL011880, 2001.

Nandy, D. and Choudhuri, A. R.: Explaining the Latitudinal Distribution of Sunspots with Deep Meridional Flow, Science, 296, 1671-1673, doi:10.1126/science.1070955, 2002.

Nandy, D., Munoz-Jaramillo, A., and Martens, P. C. H.: The Unusual Minimum of sunspot cycle 23 caused by meridional plasma flow variations, Nature, 471, 80-82, doi:10.1038/nature09786, 2011.

Oliver, R. and Ballester, J. L.: The north-south asymmetry of sunspot areas during solar cycle 22, Solar Phys., 152, 481-485, doi:10.1007/BF00680451, 1994.

Parker, E. N.: Hydromagnetic Dynamo Models, Astrophys. J., 122, 293-314, doi:10.1086/146087, 1955.

Parker, E. N.: A solar dynamo surface wave at the interface between convection and nonuniform rotation, Astrophys. J., 408, 707-719, doi:10.1086/172631, 1993.

Popova, H. P.: Effects of various types of solar meridional circulation on the propagation of dynamo waves, Astron. Rep., 53, 863-868, doi:10.1134/S1063772909090091, 2009.

Popova, H. P.: The effect of meridional circulation on dynamo waves approaching the solar poles, Astron. Rep., 54, 756-761, doi:10.1134/S1063772910080093, 2010.

Popova, H. P. and Sokoloff, D.: Meridional circulation and dynamo waves, Astron. Nachr., 329, 766-769, doi:10.1002/asna.200811028, 2008.

Popova, H. P., Reshetnyak, M. Yu., and Sokoloff, D.: Meridional circulation and dynamo-wave propagation, Astron. Rep., 52, 157-163, doi:10.1134/S1063772908020078, 2008.

Popova, H. P.: Configurations of dynamo waves in a two-layer medium and the 11-year solar cycle, Astron. Lett., 37, 210-217, doi:10.1134/S106377371101004X, 2011.
Popova, H. P., Artyushkova, M. E., and Sokoloff, D. D.: The WKB approximation for the interface dynamo, Geophys. Astrophys. Fluid Dyn., 104, 631-641, doi:10.1080/03091929.2010.507201, 2010.

Ruzmaikin, A. A., Sokolov, D. D., and Turchaninov, V. I.: The Turbulent Dynamo in a Disk, Soviet Astron., 24, 182-187, 1980.

Simpson, J. A., Zhang, M., and Bame, S.: A Solar Polar NorthSouth Asymmetry for Cosmic-Ray Propagation in the Heliosphere: The ULYSSES Pole-to-Pole Rapid Transit, Astrophys. J., 465, L69-L72, doi:10.1086/310127, 1996.

Schou, J., Antia, H. M., Basu, S., Bogart, R. S., Bush, R. I., Chitre, S. M., Christensen-Dalsgaard, J., di Mauro, M. P., Dziembowski, W. A., Eff-Darwich, A., Gough, D. O., Haber, D. A., Hoeksema, J. T., Howe, R., Korzennik, S. G., Kosovichev, A. G., Larsen, R. M., Pijpers, F. P., Scherrer, P. H., Sekii, T., Tarbell, T. D., Title, A. M., Thompson, M. J., and Toomre, J.: Helioseismic Studies of Differential Rotation in the Solar Envelope by the Solar Oscillations Investigation Using the Michelson Doppler Imager, Astrophys. J., 505, 390-417, doi:10.1086/306146, 1998.

Temmer, M., Veronig, A., and Hanslmeier, A.: Hemispheric Sunspot Numbers $R_{\mathrm{n}}$ and $R_{\mathrm{S}}$ : Catalogue and N-S asymmetry analysis, Astrophys. J., 309, 707-715, doi:10.1051/00046361:20020758, 2002.

Tobias, S. M., Cattaneo, F., and Boldyrev, S.: eprint arXiv:1103.3138, 2011.

Tuominen, J., Tuominen, I., and Kyrolainen, J.: Eleven-year cycle in solar rotation and meridional motions as derived from the positions of sunspot groups, Mon. Not. Roy. Astron. Soc., 205, 691704, 1983.

Verma, V. K.: On the north-south asymmetry of solar activity cycles, Astrophys. J., 403, 797-800, doi:10.1086/172250, 1993.

Wang, Y. M., Sheeley, N. R., and Nash, A. G.: A new solar cycle model including meridional circulation, Astrophys. J., 383, 431442, doi:10.1086/170800, 1991.

White, O. R. and Trotter, D. E.: Note on the Distribution of Sunspots Between the North and South Solar Hemispheres and its Variation with the Solar Cycle, Astrophys. J., 33, 391-392, doi:10.1086/190432, 1977.

Zhao, J. and Kosovichev, A.: Torsional Oscillation, Meridional Flows, and Vorticity Inferred in the Upper Convection Zone of the Sun by Time-Distance Helioseismology, Astrophys. J., 603, 776-784, doi:10.1086/381489, 2004.

Zeldovich, Ya. B., Ruzmaikin, A. A., and Sokoloff, D. D.: Magnetic Fields in Astrophysics, Gordon and Breach, New York, 1983.

Zharkov, S. I. and Zharkova, V. V.: Statistical analysis of the sunspot area and magnetic flux variations in 1996-2005 extracted from the Solar Feature Catalogue, Adv. Space Res., 38, 868-875, doi:10.1016/j.asr.2006.03.035, 2006.

Zharkova, V. V. and Zharkov, S. I.: Active Latitudes for Sunspot Occurrences in the Solar Cycle 23, in: Advances in Geosciences, Volume 21, Solar Terrestrial (ST), edited by: Duldig, M., World Scientific, Singapore, p. 289, 2010.

Zharkov, S. I. and Zharkova, V. V.: Statistical properties of $H_{\alpha}$ flares in relation to sunspots and active regions in the cycle 23, J. Atmos. Solar-Terr. Phy., 73, 264-270, doi:10.1016/j.jastp.2009.11.010, 2011. 
Zharkov, S. I., Gavryuseva, E., and Zharkova, V. V.: The Observed Long- and Short-Term Phase Relation between the Toroidal and Poloidal Magnetic Fields in Cycle 23, Solar Phys., 248, 339-358, doi:10.1007/s11207-007-9109-0, 2008.
Zharkova, V. V., Shepherd, S. J., and Zharkov, S. I.: Principal component analysis of background and sunspot magnetic field variations during solar cycles 21-23, Mon. Not. Roy. Astron. Soc., 424, 2943-2953, doi:10.1111/j.1365-2966.2012.21436.x, 2012. 